\title{
Allowing the Data to Speak Freely: The Macroeconometrics of the Cointegrated Vector Autoregression
}

16 November 2007

Corresponding Author:

Kevin D. Hoover

Department of Economics

Duke University

Box 90097

Durham, NC 27278

U.S.A.

Tel. $+(919) 660-1876$

Fax $+(919)$ 684-8974

E-mail

kd.hoover@duke.edu
Søren Johansen

Department of Economics

University of Copenhagen

Studiestræde 6

1455 København K

Denmark

Tel. +4535320681

Fax +45 35320772

Email

soren.johansen@econ.ku.dk
Katarina Juselius

Department of Economics

University of Copenhagen

Studiestræde 6

1455 Copenhagen K

Denmark

Tel. +45-35323068

Fax +45-35 323064

E-mail

katarina.juselius@econ.ku.dk

Session Title: Complexity and Dynamics in Macroeconomics: Alternatives to the DSGE Models

Session Chair: David Colander, Middlebury College

Discussants: Stephen Turnovsky, University of Washington

Duncan Foley, New School University 


\section{Abstract \\ of \\ Allowing the Data to Speak Freely: The Macroeconometrics of the Cointegrated Vector Autoregression}

An explication of the key ideas behind the Cointegrated Vector Autoregression Approach. The CVAR approach is related to Haavelmo's famous "Probability Approach in Econometrics" (1944). It insists on careful stochastic specification as a necessary groundwork for econometric inference and the testing of economic theories. In time-series data, the probability approach requires careful specification of the integration and cointegration properties of variables in systems of equations. The relationship between the CVAR approach and wider methodological issues and between it and related approaches (e.g., the LSE approach) are explored. The specific-to-general strategy of widening the scope of econometric models to identify stochastic trends and cointegrating relations and to nest theoretical economic models is illustrated with the example of purchasing-power parity.

Keywords: cointegrated vector autoregression, VAR, CVAR, cointegration, Johansen, Juselius, Haavelmo, time-series models, Walrasian methodology, Marshallian methodology

JEL Codes: B41, C30, C32, C50, C51, C52 
All economists agree that reality is complex and that the tools with which we confront it are far simpler. Theorists sometimes deal with this gap by asking very little of the data. Start with the "stylized" facts and develop relatively simply theories to account for them. Unfortunately, stylized facts are often too stylized to discriminate among plausible candidate theories or to provide a basis for accurate quantification. Alternative approaches start from the other end and ask much of the data. One European tradition, which derives from Trygve Haavelmo's “The Probability Approach in Econometrics" (1944), focuses on obtaining good characterizations of data before testing and on drawing out the implications of data that ought to constrain economic theorizing. The application of the cointegrated vector autoregression (CVAR) recounted, for example, in Katarina Juselius's (2006) textbook and facilitated by the CATS in RATS econometric software (Jonathan G. Dennis et al., 2006) is a special macroeconometric case of the Probability Approach. The message of the Probability Approach and the CVAR approach can be summarized in the slogan: "facts, not stylized facts."

\section{Between Data and Theory}

All econometrics aims ultimately to confront theory and data. Different approaches differ in how they conceive the relationship and the problems that it poses. To start, think of an ideal case such as one might find in a physics textbook. The law of gravity is applied to the dropping of a ball from a tower. The law, together with an initial condition (the height of the tower), determines the distance the ball falls for each time ... in theory. Of course, no object conforms perfectly to the gravity law. If one had a generous enough notion of approximation, if the ball were steel and the initial height were not too high, then tight bounds of approximation would work; but not if the ball were styrofoam. Now there are three choices: A) declare that theory is no good; B) modify the original 
theory to account for the factors such as air resistance; or C) attempt to assess empirically the combined forces that must be used to adjust the gravity law to its application in the particular case.

In economics, as in physics, the difficulty is that our theory holds ceteris paribus. When other things are not equal, there is always some residual left unexplained which, if large, may render the theory empirically irrelevant. As scientists, we can either attempt to elaborate the theory in such a way that fewer and fewer ceteris paribus conditions are invoked (B) or we can attempt to provide an adequate empirical characterization of the factors that determine the initial gap between theory and data (C). Strategy $\mathrm{C}$ can be seen as the passive analogue to a controlled experiment (cf. Haavelmo, 1944, esp. chaps. 1 and 2). It has an advantage over B, in the sense of providing clues as to how the theory needs to be developed - clues that would be helpful in strategy B, but for which strategy B itself offers no internal resources. What is more, strategy $\mathrm{C}$ gives some hope of actually isolating the action of the gravity law and, therefore, in fact testing whether it is a contributing factor to a successful account of the data. It is only when we can control for enough of the complicating factors that the underlying quadratic relationship of the gravity law can be detected.

The extreme limit of strategy B is what Milton Friedman has called the "Walrasian" methodology, by which he means not general-equilibrium theory per se, but to the idea that one most have a complete, detailed theoretical account in order to say anything useful about the economy at all (see Kevin D. Hoover, 2006). The extreme limit of strategy $\mathrm{C}$ is a completely atheoretical analysis of data. Both extremes are hopeless: strategy B because we lack the cognitive capacity to elaborate a complete theory from first principles; strategy $\mathrm{C}$ because without some prior conceptual notion we would never find a starting place for any investigation. Still, the Probability Approach leans toward strategy $\mathrm{C}$ : the weight of the analysis is on characterizing the data and on using the data to criticize and guide theorizing. In Friedman's terms, the approach is "Marshallian" or, as Hoover (2006) puts 
it, "archaeological": we learn about the economy a piece at a time by removing the overlay of detritus to uncover the underlying structure, guided by our theoretical conception of what we are looking for, which is tested and enriched by each new discovery.

\section{Models of Theory, Models of Data}

The CVAR approach builds on Haavelmo's (1944) great insight that the gap between theory and data need not be treated as an unstructured residual of approximation but could itself be modeled statistically using the theory of probability. The cost is that we now need another level of modeling in addition to theory - a statistical model constructed in such a way that i) theory has implications interpretable in its terms and ii) data are described fully enough that its only residuals are identically independent random errors - that is, unsystematic noise. The payoff is that such a statistical model warrants the use of likelihood methods and provides a firm basis for deductions about the implications for theory.

The CVAR approach sees the world as a highly complex dynamic system, the properties of which must be inferred from data reflecting a single (nonreplicable) realization of a multivariate, path-dependent process. Naturally, this data-generating process must be approximated by simpler relationships, which characterize the data accurately enough for our particular purposes. The statistical model ties economic theory to the data when it nests both the data-generating process and the theoretical model. Then the parameters of the theoretical model can be read as assertions about parameters of the statistical model, which can be tested against the data provided that the statistical model characterizes it accurately.

While we can never know for certain that our statistical model captures the data-generating process, we can often find compelling evidence when it does not. Søren Johansen (2006, pp. 293295) provides an example, which starts with the unobservable data-generating process: 


$$
x_{t}=0.9 x_{t-1}+1.0+\varepsilon_{t}, t=1,2, \ldots, 100, x_{0}=10,
$$

where the $\varepsilon_{t}$ are identically independently distributed (i.i.d) $N(0,1)$. Note that $E\left(x_{t}\right)=1 /(1-0.9)=10$ and $\operatorname{var}\left(x_{t}\right)=1 /\left(1-0 \cdot 9^{2}\right)$. Consider an economic theory that predicts that the mean value of $x$ is $\mu=10$ (it happens that our theory is exactly true, but that will not generally be the case). To test the theory we need to provide a model of the probability process. One model is

$$
x_{t}=\mu+\varepsilon_{t},
$$

where the $\varepsilon_{t}$ are i.i.d. $N\left(0, \sigma^{2}\right)$. Omitting details, the maximum likelihood estimates of (2) yield a 95 percent asymptotic confidence interval of $\hat{\mu} \pm 1.96 \hat{\sigma} / \sqrt{T}=9.138 \pm 0.449$. Since 10 does not lie within the confidence interval, it might appear, then, that we have good grounds to reject the hypothesis that $\mu=10$. Of course, that claim is only as good as the probability model in which it is based. Model (2) models the errors terms as i.i.d normal. Given the data-generating process (1), this assumption will be violated; a simple statistical test would show that the estimated residuals are serially correlated. We can conclude, then, that the data-generating process is not nested in our statistical model, that the estimates are not reliable, and that the theory has not been tested adequately.

An alternative statistical model is given by

$$
x_{t}=\rho x_{t-1}+\mu(1-\rho)+\varepsilon_{t},
$$

where again the $\varepsilon_{t}$ are i.i.d. $N\left(0, \sigma^{2}\right)$ and $E\left(x_{t}\right)=\mu$, if $|\rho|<1$. Now, as it happens, the datagenerating process (1) is precisely nested in (3). Again omitting details, the maximum likelihood estimate of (2) yields a 95 percent asymptotic confidence interval of $\hat{\mu} \pm 1.96 \hat{\sigma} /[(1-\rho) \sqrt{T}]=9.123 \pm 2.247$. The previous estimate of $\mu$ was spuriously precise. On the current probability model (3), the estimate is less precise and we cannot reject $\mu=10$. 
We cannot have sufficient conditions for knowing that the data-generating process is nested in any conjectured probability model. One message of our cooked example is that we should check the necessary conditions. Not only did this lead us to reject (2) as an adequate description of the probability model, the serial correlation of the residuals from estimating (2) naturally suggest models in the autoregressive class such as (3). Another message is that our tests of theory will go seriously wrong if we base them on statistical models that fail accurately to characterize the data in important ways. The upshot of these messages is that key elements of the CVAR approach are, first, to get the probability model right, which is judged by ruthless application of diagnostic testing, and second, to judge theories in relation to the testable restrictions that they imply for the probability model.

Accurately characterizing the persistence of the data is a vital aspect the CVAR approach. In equation (3), we assumed that $|\rho|<1$, which implies that the $x_{t}$ is mean-reverting or stationary - i.e., integrated of order zero or I( 0$)$. But if instead, $\rho=1$, then $x_{t}$ would be a nonstationary, unit-root process (i.e., I(1)) in which shocks would accumulate, forming a stochastic trend (i.e., a permanent, nondeterministic shift in the mean). Statistical inferences that fail to account for nonstationarity (deterministic or stochastic) will be misleading in a manner analogous to inferences based on equation (2). One way to account for a unit root is to transform the data to stationarity by differencing. But differencing throws away all the long-run information in the data. Fortunately, when data share a stochastic trend, a particular linear combination of the levels of the variables will also be stationary. Such variables are said to be cointegrated. Cointegration was formalized by Robert F. Engle and Clive W. J. Granger (1987), although it is implicit in the earlier work of the London School of Economics (LSE) approach (see Grayham E. Mizon, 1995). Since economic theories frequently have clear, testable implications about degrees of persistence and cointegration, these dynamic properties are central to the CVAR approach. For example, a stationary cointegrating 
relationship among nonstationary variables can frequently be interpreted as defining a long-run equilibrium toward which variables are adjusting. This is the famous "error-correction mechanism" of the LSE approach.

\section{The Cointegrated Vector Autoregression Model}

The CVAR provides a simple linear system that can characterize the probability distribution of a set of variables. While the importance of cointegration is widely accepted, the CVAR approach can be distinguished from its close ally, the LSE approach, which has most often focused on singleequations, even though it can be readily generalized to systems (David F. Hendry, 1995). And the CVAR approach can be distinguished from many other applications of cointegration in systems of equations by its focus on well-specified, congruent statistical models - a hallmark of Haavelmo's probability approach. Recognition that macroeconomic time-series data are typically nonstationary and cointegrated motivated, first, the development of likelihood-based inference for the CVAR model (Johansen, 1996), including tests for determining the numbers of stochastic trends and cointegrating relations and tests of hypotheses on their structure and an applied macroeconometric methodology (Juselius, 2006), a practical method of asking within the broad context of a theoretical model, what do the data say when they are allowed to speak freely? The necessary tests and methods are implemented in CATS in RATS (Dennis et al., 2006).

Two facts argue for the CVAR approach: first, statistical evidence indicates that nonstationary data are pervasive; second, economic theory is mainly about the adjustment of one variable to another in search of individually optimal, systemically more coordinated outcomes. By combining differenced and cointegrated data, the CVAR model responds to both facts. Economic data are analyzed as short-run variations around moving longer run equilibria. Longer run forces are themselves divided into the forces that move the equilibria (pushing forces, which give rise to 
stochastic trends) and forces that correct deviations from equilibrium (pulling forces, which give rise to cointegrating relations). Interpreted in this way, the CVAR has a good chance of nesting a multivariate, path-dependent data-generating process and relevant dynamic macroeconomic theories. Unlike approaches in which the data are silenced by prior restrictions, the CVAR model gives the data a rich context in which to speak freely.

Especially with respect to persistence properties, it is worth recalling that the CVAR is not the underlying data-generating process; rather it is a good enough approximation for a particular problem. For example, we can (and usually should) approximate highly persistent data by an exact unit root, since tests based on $\chi^{2}-, F$-, and $t$-distributions and the assumption of stationarity will go badly wrong when the data-generating process has a near unit root, unless we have a very long sample of, say, more than 5000 observations (Johansen, 2006).

Much of the focus in the CVAR approach is on the long run: can we identify the stochastic shocks? what are the cointegrating relations? Juselius and Massimo Franchi (2007; also Johansen, 2006) show how to translate the assumptions underlying a dynamic, stochastic generalequilibrium (DSGE) model (Peter N. Ireland, 2004) into a set of testable assumptions on cointegrating relationships and stochastic trends in a CVAR. Accounting for (near) unit roots in the model provides a powerful tool to robustify statistical and economic inference. Most assumptions underlying the dynamic, stochastic general-equilibrium model and, hence, the real business cycle model were rejected when properly tested. Structuring the data in this way offers a number of facts, for example that it was shocks to consumption that have generated the long business cycles, that a theory model should replicate in order to claim empirical relevance. Thus, the CVAR approach provides both a critical framework and constructive insights. 
Johansen et al.'s (2007) investigation of purchasing-power parity provides a more detailed case study. Call the logarithm of German prices $p_{1}$, the logarithm of U.S. prices $p_{2}$, and the logarithm of the exchange rate $s_{12}$. Inflation rates $\left(\Delta p_{1}, \Delta p_{2}\right)$ display a stochastic trend; and, since inflation rates are I(1), price levels are I(2). The exchange rate is I(1). Since prices are a higher level of integration than exchange rates, purchasing-power parity requires that prices be cointegrated - that is, relative prices must be I(1) $\left(p p=p_{1}-p_{2} \sim \mathrm{I}(1)\right)$ and exchange rates and relative prices must share a common trend, so that $p p p=p_{1}-p_{2}-s_{12} \sim \mathrm{I}(0)$. Then, the hypothesis of purchasing-power-parity in the dollar/deutschmark case amounts to a complex hypothesis: $\left\{p_{1} \sim \mathrm{I}(2), p_{2} \sim \mathrm{I}(2), p p=p_{1}-p_{2} \sim \mathrm{I}(1)\right.$, $s_{12} \sim \mathrm{I}(1), p p p=p_{1}-p_{2}-s_{12} \sim \mathrm{I}(0), p_{1} \& p_{2}$ are pushing, $s_{12}$ is adjusting\}. Here "adjusting" means that the shocks to the nominal exchange rate do not contribute to any stochastic trend, even the ones that drive $s_{12}$ itself. A careful CVAR analysis, confirms that $p_{1} \sim \mathrm{I}(2), p_{2} \sim \mathrm{I}(2), p p \sim \mathrm{I}(1)$, and $s_{12} \sim$ I(1); but, contrary to the hypothesis, $p p$ and $s_{12}$ have different stochastic trends, so that they are not cointegrating (i.e., $p p p \sim \mathrm{I}(1)$ ), and $s_{12}$ is pushing.

The failure of purchasing-power parity requires that we dig deeper. General equilibrium implies that a persistent departure from purchasing-power parity must generate a similar persistent movement somewhere else in the economy. Since exchange rates are involved in capital movements as well as trade in goods and services, a natural place to look is in the behavior of interest rates. Adding the interest rates on German and U.S. long-term bonds $\left(b_{1}\right.$ and $\left.b_{2}\right)$ generalizes the more specific original model. In the more general model, the I(1) trend in ppp is the same as the trend in the relative bond yield $\left(b_{1}-b_{2}\right)$, so that ppp and the real interest-rate differential are cointegrating: $\left(p p p-\omega\left[\left(b_{1}-\Delta p_{1}\right)-\left(b_{2}-\Delta p_{2}\right)\right] \sim \mathrm{I}(0)\right.$, where $\omega$ is a constant parameter. Such a "specific-togeneral approach," which starts with a small model and works to a larger one, is justified because cointegration is a property that is invariant to widening the data set. 
How should one understand these findings economically? While it is beyond our present purpose to make a detailed case, the failure of purchasing-power parity appears to be related to the joint determination of nominal exchange rates in the goods and the foreign-exchange market, and how the latter influences determination of interest rate. These findings are consistent with the application to the foreign-exchange market of the theory of imperfect knowledge economics as

developed by Roman Frydman and Michael D. Goldberg (2007). Such a theory is post-Walrasian in the sense that it rejects a central tenet of modern Walrasian macroeconomics - the rational expectations hypothesis. But more fundamentally, it is post-Walrasian (that is Marshallian in Friedman's sense or archaeological in Hoover's) in that it rejects the privileging of a priori economic theory over empirical evidence. In the language of the CVAR approach, empirical evidence is the pushing force and economic theory is adjusting.

\section{References}

Colander, David, ed. Post Walrasian macroeconomics: Beyond the dynamic stochastic general equilibrium model. Cambridge: Cambridge University Press, 2006.

\section{Dennis, Jonathan G.; Juselius, Katarina; Johansen, Søren; and Hansen, Henrik. CATS in} RATS: Cointegration analysis of time series, software. Evanston, IL: Estima, 2006.

Frydman, Roman and Goldberg, Michael D. Imperfect knowledge economics: Exchange rates and risk. Princeton: Princeton University Press, 2007.

Engle, Robert F. and Granger, Clive W. J. "Co-integration and Error Correction: Representation, Estimation, and Testing," Econometrica, 1987, 55(2), pp. 251-76

Haavelmo, Trygve. "The Probability Approach in Econometrics," Econometrica, 1944, 12(supplement).

Hendry, David F. Dynamic Econometrics. Oxford: Oxford University Press, 1995. 
Hoover, Kevin D. "The Past as Future: The Marshallian Approach to Post Walrasian Econometrics," in Colander (2006), pp. 239-57.

Ireland, Peter N. "A Method for Taking Models to the Data." Journal of Economic Dynamics \& Control, 2004, 28(6), pp. 1205-26.

Johansen, Søren. Likelihood-based inference in cointegrated vector autoregressive models. Oxford: Oxford University Press, 1996.

Johansen, Søren. "Confronting the Economic Model with the Data," in Colander (2006), pp. 287300.

Johansen, Søren; Juselius, Katarina; Frydman, Roman; and Goldberg, Michael D. "Testing Hypotheses in an I(2) Model with Applications to the Persistent Long Swings in the Dmk/\$ Rate," unpublished manuscript, 2007.

Juselius, Katarina. The cointegrated VAR model. Oxford: Oxford University Press, 2006.

Juselius, Katarina and Franchi, Massimo. "Taking a DSGE Model to the Data Meaningfully." Economics-The Open-Access, Open-Assessment E-Journal 2007, No. 2007-4, http://www.economics-ejournal.org/economics/journalarticles/2007-4.

Juselius, Katarina and Johansen, Søren. "Extracting Information from the Data: A European View on Empirical Macro," in Colander (2006), pp. 301-334.

Mizon, Grayham E. "Progressive Modeling of Macroeconomic Time Series: The LSE Methodology," in Kevin D. Hoover, ed., Macroeconometrics: Developments, tensions and prospects. Boston: Kluwer, 1995, pp. 107-170. 